\title{
Simultaneous determination of major type A and B trichothecenes, zearalenone and certain modified metabolites in Finnish cereal grains with a novel liquid chromatography-tandem mass spectrometric method
}

\author{
Alexis V. Nathanail ${ }^{1} \cdot$ Jenna Syvähuoko $^{1} \cdot$ Alexandra Malachová $^{2} \cdot$ Marika Jestoi $^{3}$ • \\ Elisabeth Varga ${ }^{2} \cdot$ Herbert Michlmayr ${ }^{4}$ - Gerhard Adam ${ }^{4}$ - Elina Sieviläinen ${ }^{5}$. \\ Franz Berthiller ${ }^{2} \cdot$ Kimmo Peltonen $^{6}$
}

Received: 5 February 2015 /Revised: 31 March 2015 / Accepted: 2 April 2015 / Published online: 3 May 2015

(C) The Author(s) 2015. This article is published with open access at Springerlink.com

\begin{abstract}
A reliable and sensitive liquid chromatographytandem mass spectrometric method was developed for the simultaneous quantitative determination in cereals of the Fusarium mycotoxins HT-2 toxin, T-2 toxin, deoxynivalenol, nivalenol and zearalenone, as well as the modified metabolites 3 -acetyl-deoxynivalenol, $\alpha$-zearalenol, $\beta$-zearalenol, deoxynivalenol-3-glucoside, HT-2-3-glucoside, nivalenol-3glucoside, zearalenone-14-glucoside, zearalenone-14-sulphate, zearalenone-16-glucoside, $\alpha$-zearalenol-14-glucoside
\end{abstract}

Electronic supplementary material The online version of this article (doi:10.1007/s00216-015-8676-4) contains supplementary material, which is available to authorized users.

Alexis V. Nathanail

alexis.nathanail@helsinki.fi

1 Chemistry and Toxicology Unit, Research and Laboratory Department, Finnish Food Safety Authority (Evira), Mustialankatu 3, 00790 Helsinki, Finland

2 Christian Doppler Laboratory for Mycotoxin Metabolism and Center for Analytical Chemistry, Department for Agrobiotechnology (IFATulln), University of Natural Resources and Life Sciences, Vienna (BOKU), Konrad Lorenz Str. 20, 3430 Tulln, Austria

3 Product Safety Unit, Control Department, Finnish Food Safety Authority (Evira), Mustialankatu 3, 00790 Helsinki, Finland

4 Department of Applied Genetics and Cell Biology, University of Natural Resources and Life Sciences, Vienna (BOKU), Konrad Lorenz Str. 24, 3430 Tulln, Austria

5 Plant Analysis Unit, Research and Laboratory Department, Finnish Food Safety Authority (Evira), Mustialankatu 3, 00790 Helsinki, Finland

6 Finnish Safety and Chemicals Agency (Tukes), Opastinsilta 12, 00521 Helsinki, Finland and $\beta$-zearalenol-14-glucoside. The 'dilute and shoot' approach was used for sample preparation after extraction with acetonitrile:water:acetic acid (79:20:1, v/v/v). Separation was carried out using reversed-phase liquid chromatography, and detection was performed using tandem mass spectrometry in the selected reaction monitoring mode. The method was inhouse validated according to performance characteristics, established in Commission Regulation EC No 401/2006 and Commission Decision EC No 657/2002, prior to its application in a nationwide survey for the analysis of barley, oat and wheat samples $(n=95)$ harvested in Finland during 2013. Deoxynivalenol and its glucosylated form were the most abundant of the analytes, being detected in 93 and $81 \%$ of the samples, respectively. Concentrations of deoxynivalenol were unusually high in 2013, especially in oats, with some cases exceeding the maximum legislative limits for unprocessed oats placed on the market for first-stage processing. All modified mycotoxins analysed were detected, and the natural occurrence of some of these compounds (e.g. zearalenone-16-glucoside and nivalenol-3-glucoside) in barley, oats and/or wheat was documented for the first time.

Keywords Fusarium mycotoxins · Masked mycotoxins · LC-MS/MS · Method validation · Survey

\section{Introduction}

Plant pathogenic species of the genus Fusarium are widespread pathogens of small-grain cereals, capable of producing an array of heterogeneous mycotoxins. These fungal secondary metabolites exert a broad range of biological activities that may cause acute or chronic health problems in humans and 
animals after the consumption of mycotoxin-contaminated commodities. In northern Europe, the most important fusariotoxins in cereal grains are trichothecenes and zearalenone (ZEN) [1].

To date, more than 200 trichothecenes have been identified [2] and are classified according to their structure into four types: $\mathrm{A}, \mathrm{B}, \mathrm{C}$ and D [3]. These compounds share a common cyclic sesquiterpene skeleton with a C-12, C-13 epoxy ring (Fig. 1A). Manifestations of toxic effects following exposure to trichothecenes include haematotoxicity, neurotoxicity and immunosuppression, causing animal feed refusal, growth retardation and vomiting $[4,5]$. Toxins belonging to the type A group, such as HT-2 toxin (HT2) and T-2 toxin (T2), as well as the type B trichothecenes deoxynivalenol (DON) and nivalenol (NIV) are relevant to food safety due to their occurrence in edible crops. According to survey data from Finland and other Nordic countries, oats are usually more severely affected by high DON and HT2/T2 concentrations compared to barley and wheat [6]. During the past decade, high incidences of HT2 and T2 have been documented in raw oats from several northern European countries, with the mean concentrations varying considerably from year to year (e.g. [7, 8]). For NIV, a negative relationship with the abundance of high DON levels has been observed, which can mainly be attributed to the production of these mycotoxins by different Fusarium species [9].

ZEN is a macrocyclic $\beta$-resorcyclic acid lactone consisting of a ring with two ketones and an aromatic ring substituted with two hydroxyl groups (Fig. 1B). ZEN and its phase I metabolites, $\alpha$-zearalenol ( $\alpha$-ZEL) and $\beta$-zearalenol $(\beta$ ZEL), exert strong oestrogenic effects on vertebrates by binding to the oestrogen receptors ER- $\alpha$ and ER- $\beta$ [10].
Subchronic and long-term toxicity experiments have confirmed the oestrogenic activity of ZEN in livestock, causing infertility, reduced foetal weight or embryonic death [11]. Oats have been found to contain the highest ZEN concentrations in Finland (up to $1690 \mu \mathrm{g} / \mathrm{kg}$ ) according to survey data obtained during the period 1999-2009 [9].

Trichothecenes and ZEN, similarly to other xenobiotics, are metabolised in living organisms by natural detoxification mechanisms into products distinct from the native toxins. These compounds may be activated versions of the native form as a result of phase I metabolism, or phase II conjugated metabolites with glucose, sulphate or amino acid moieties, and are defined as 'modified mycotoxins' [12]. The term 'masked mycotoxins', which was originally used to describe altered forms of mycotoxins, henceforth refers exclusively to plantgenerated mycotoxin metabolites [13]. Compounds formed during food manufacturing processes or metabolites excreted directly by fungi (e.g. 3-acetyl-deoxynivalenol, 3Ac-DON) are also classified as modified mycotoxins. Several in planta trichothecene conjugates have already been reported, including deoxynivalenol-3-glucoside (DON3Glc) [14], HT-2-3glucoside (HT2-3-Glc) [15] and nivalenol-3-glucoside (NIV3Glc) [16]. In addition to the major ZEN metabolites, the ZELs, other plant-specific metabolites, have been described, such as zearalenone-14-glucoside (ZEN14Glc), $\alpha$ ZEL-14-glucoside ( $\alpha$-ZEL14Glc), $\beta$-ZEL-14-glucoside $(\beta$ ZEL14Glc), ZEN-14-sulphate (ZEN14Sulf) [17] and the recently reported zearalenone-16-glucoside (ZEN16Glc) [18]. From a toxicological perspective, DON3Glc and ZEN14Glc are the most studied plant-generated modified mycotoxins, and according to current knowledge, their inherent toxicity

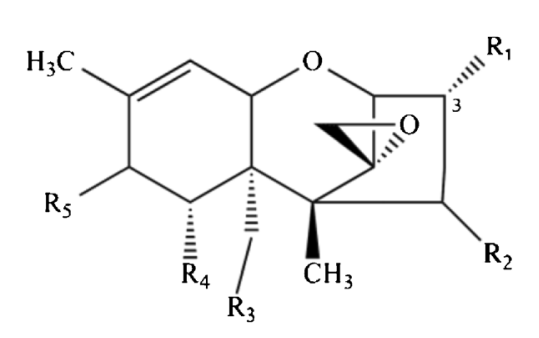

\begin{tabular}{|c|c|c|c|c|c|}
\hline Analyte & $R_{1}$ & $R_{2}$ & $\mathrm{R}_{3}$ & $\mathrm{R}_{4}$ & $R_{5}$ \\
\hline HT2 & $\mathrm{OH}$ & $\mathrm{OH}$ & $\mathrm{OCOCH}_{3}$ & $\mathrm{H}$ & $\mathrm{OCOCH}_{2} \mathrm{CH}\left(\mathrm{CH}_{3}\right)_{2}$ \\
\hline HT2-3-Glc & $\mathrm{OC}_{6} \mathrm{H}_{11} \mathrm{O}_{5}$ & $\mathrm{OH}$ & $\mathrm{OCOCH}_{3}$ & $\mathrm{H}$ & $\mathrm{OCOCH}_{2} \mathrm{CH}\left(\mathrm{CH}_{3}\right)_{2}$ \\
\hline & $\mathrm{OH}$ & $\mathrm{OCOCH}_{3}$ & $\mathrm{OCOCH}_{3}$ & $\mathrm{H}$ & $\mathrm{OCOCH}_{2} \mathrm{CH}\left(\mathrm{CH}_{3}\right)_{2}$ \\
\hline 3AC-DON & $\mathrm{OCOCH}_{3}$ & $\mathrm{H}$ & $\mathrm{OH}$ & $\mathrm{OH}$ & $=0$ \\
\hline DON & $\mathrm{OH}$ & $\mathrm{H}$ & $\mathrm{OH}$ & $\mathrm{OH}$ & $=0$ \\
\hline DON3Glc & $\mathrm{OC}_{6} \mathrm{H}_{11} \mathrm{O}_{5}$ & $\mathrm{H}$ & $\mathrm{OH}$ & $\mathrm{OH}$ & $=0$ \\
\hline NIV & $\mathrm{OH}$ & $\mathrm{OH}$ & $\mathrm{OH}$ & $\mathrm{OH}$ & $=0$ \\
\hline NIV3GIC & $\mathrm{OC}_{6} \mathrm{H}_{11} \mathrm{O}_{5}$ & $\mathrm{OH}$ & $\mathrm{OH}$ & $\mathrm{OH}$ & $=0$ \\
\hline
\end{tabular}

Abbreviations: HT2, HT-2 toxin; HT2-3-Glc, HT-2-3-glucoside; T2, T-2 toxin; 3AC-DON, 3-acetyl-deoxynivalenol; DON, deoxynivalenol; DON3Glc, deoxynivalenol-3-glucoside; NIV, nivalenol; NIV3GIc, nivalenol-3-glucoside.

(A)

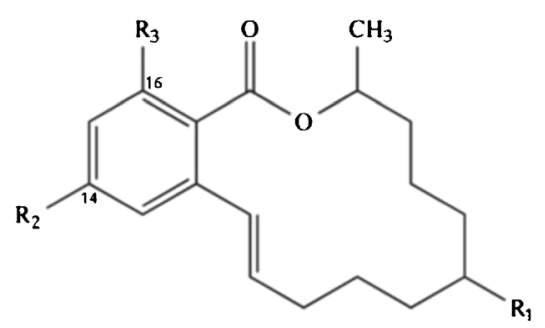

(B)

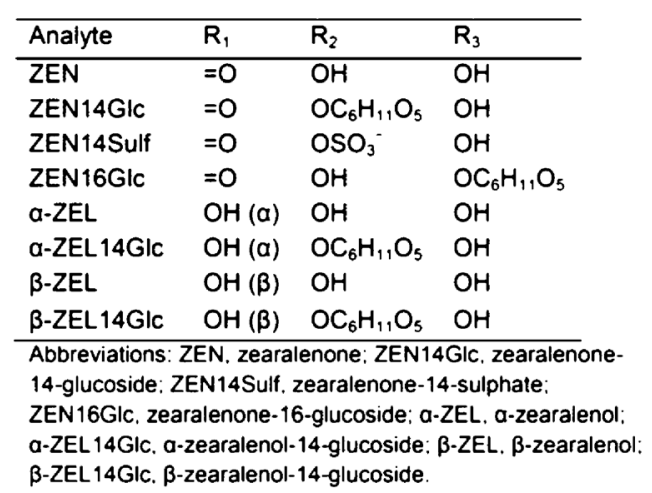

Fig. 1 Chemical structures of native and modified type A and B trichothecenes (A) and zearalenone (B) 
is lower than that of their respective native forms. However, toxicological implications may arise from the reactivation of these compounds by the action of intestinal microbiota during mammalian digestion $[19,20]$.

In order to conduct a comprehensive exposure assessment concerning modified mycotoxins and investigate the multicomponent nature of mycotoxin-contaminated samples, improved analytical methodologies are necessary. The majority of modern analytics for mycotoxin quantification in cereals and cereal-derived foodstuffs rely on liquid chromatographytandem mass spectrometry (LC-MS/MS) [21]. LC-MS-based methods can render multiple native mycotoxins and potentially also conjugated forms directly accessible for detection [22]. However, methods that have initially been developed for determination of parent mycotoxins may suffer from inherent problems (e.g. poor recovery of polar analytes), chromatographic deficiencies or inadequate sensitivity when used for analysis of modified mycotoxins [23]. During the past few years, dedicated multi-analyte LC-MS/MS methods have been developed for modified mycotoxins [24, 25], although these are not applicable in the majority of laboratories due to the lack of analytical standards required for their accurate measurement. Such methods, as with most multi-mycotoxin methods, have been based on the 'dilute and shoot' approach, since no clean-up has been found suitable to accommodate the wide range of analyte polarities. Recently, on-line clean-up was successfully applied for DON and DON3Glc determination, overcoming limitations related to the dilute and shoot approach, such as severe matrix effects and low sensitivity [26].

In 2006, the European Commission (EC) set maximum levels (MLs) for 11 mycotoxins, including DON and ZEN, in certain foods intended for human consumption (Commission Regulation EC No 1881/2006 [27]). Regarding T2 and HT2, so-called indicative levels for monitoring the sum of these Fusarium mycotoxins in cereals and cerealderived products were published in Commission Recommendation 2013/165/EU [28]. The same document also highlights the need for data on the occurrence of conjugated mycotoxins. The European Food Safety Authority (EFSA) recently published a scientific opinion on the risks to human and animal health related to the presence of modified forms in food and feed, addressing the need for properly validated and sensitive analytical methods [29]. In this context, the aim of our study was to develop a reliable and sensitive LC-MS/MS method for the determination of T2, HT2, DON, NIV and ZEN, as well as several of their modified metabolites in cereal grains. The method was in-house validated for barley, oats and wheat according to available legislative method performance criteria $[30,31]$ and was used to examine the occurrence of native and modified mycotoxins on Finnish grain samples.

\section{Materials and methods}

\section{Reagents and standards}

HPLC-grade acetonitrile, methanol, acetic acid and hexane were purchased from J.T. Baker (Deventer, the Netherlands). Ammonium formate (MS grade) and formic acid (p.a. grade) were obtained from Sigma-Aldrich (Steinheim, Germany), whereas ammonium acetate (MS grade) was purchased from VWR International (Leuven, Belgium). Water was purified with a Milli-Q Plus system (Millipore, Espoo, Finland). Analytical standards for HT2, T2, 3Ac-DON, DON, NIV, ZEN, $\alpha$ ZEL and $\beta$-ZEL were obtained from Sigma-Aldrich in solid form. The modified mycotoxins DON3Glc [32], ZEN14Glc, $\alpha$-ZEL14Glc, $\beta$-ZEL14Glc [33] and ZEN16Glc [18], as well as ZEN14Sulf [34], HT2-3-Glc and NIV3Glc (Michlmayr et al., in preparation), were produced by us at the University of Natural Resources and Life Sciences, Vienna (BOKU). All native mycotoxins used for the production of modified forms were purchased as analytical standards in solid form from Romer Labs (Tulln, Austria). All production procedures ended with isolation of the modified toxins by preparative HPLC under reversed-phase conditions. Their identity and purity $(>98 \%)$ were verified by NMR and LC-UV measurements. Individual stock solutions for all analytes were prepared by dissolving the solid substance in acetonitrile. In total, eight working solutions were prepared on a weekly basis by diluting the stock solutions to the appropriate concentrations. The solutions were stored at $-20^{\circ} \mathrm{C}$ and were brought to room temperature in the dark before use.

\section{Survey samples}

Spring wheat, oat and barley samples from the quality monitoring programme of the Finnish grain harvest were analysed for Fusarium mycotoxins and their in planta metabolites. The quality monitoring programme is based on grain samples sent in by farmers. The survey samples in this study $(n=95)$ represented commonly cultivated small-grain cereal varieties in Finland from different geographic areas and were randomly chosen from a total of 1117 samples of the 2013 harvest (Fig. 2). Areas 14 and 15 are not represented in this survey, since no significant cereal production takes place there. According to the background information provided by farmers, $77 \%$ of the spring wheat samples were intended for the food industry, $13 \%$ for feed and $10 \%$ for seed. Half of the barley samples were malting varieties, $38 \%$ were feed varieties and $12 \%$ were cultivated for other purposes. Concerning oats, $16 \%$ were intended for food and $84 \%$ for feed, farm trade and seed.

The samples analysed during the survey consisted of several subsamples collected from arbitrary points in each field ( $2 \mathrm{~kg}$ per sample). After harvest, the samples were air-dried to a moisture content below $15 \%$ to avoid fungal growth. Prior 


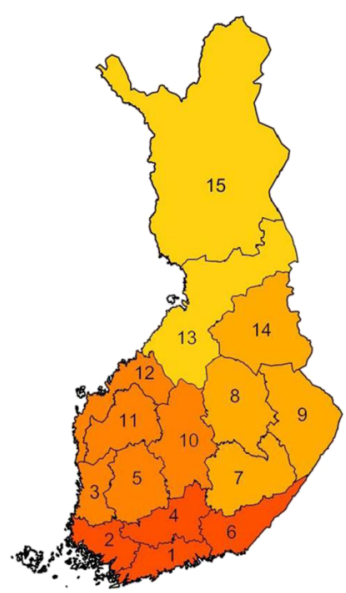

\begin{tabular}{llll}
\hline \multirow{2}{*}{ Area } & \multicolumn{3}{c}{ Cereal grains $(\boldsymbol{n}=95)$} \\
\cline { 2 - 4 } & Barley & Oats & Wheat \\
\hline 1 & 4 & 3 & 6 \\
2 & 10 & 1 & 5 \\
3 & 2 & 1 & 5 \\
4 & 5 & 3 & 3 \\
5 & 2 & 1 & 3 \\
6 & 0 & 2 & 4 \\
7 & 2 & 2 & 0 \\
8 & 0 & 3 & 1 \\
9 & 1 & 2 & 0 \\
10 & 0 & 3 & 0 \\
11 & 5 & 6 & 2 \\
12 & 3 & 1 & 0 \\
13 & 0 & 3 & 1 \\
14 & 0 & 0 & 0 \\
15 & 0 & 0 & 0 \\
\hline Total: & 34 & 31 & 30 \\
\hline \multicolumn{4}{c}{} \\
\hline
\end{tabular}

Fig. 2 Origin of the cereal samples analysed during the survey study. The colours represent the different regions: North, East, South and West

to analysis, each 2-kg sample was finely ground in its entirety using a Retsch ZM 200 grinder (Retsch GmbH, Haan, Germany) and stored at room temperature in plastic containers.

The growing period of 2013 was warm in Finland, with the average mean daily temperatures being $1.0-2.0^{\circ} \mathrm{C}$ above the long-term average $\left(17.6{ }^{\circ} \mathrm{C}\right)$ throughout the country. The spring sowing started with dry weather conditions, but during the growing season, there were regional variations in rainfall levels. Precipitation was less than a third of the long-term average of $190 \mathrm{~mm}$ in the southwestern parts of Finland, in contrast to the eastern parts, where it was generally higher than the long-term average $(>250 \mathrm{~mm})$. During the harvest time, the weather was generally dry. The meteorological data of the study were obtained from the Internet services of the Finnish Meteorological Institute (http://www.fmi.fi).

\section{Spiking experiments and sample preparation}

For the wheat and barley spiking experiments, commercially available flours were used as a blank matrix. In the case of oats, oat kernels received from the Finnish grain quality monitoring programme were used. The kernels were finely ground with a Retsch ZM 200 grinder and analysed for use as blank material by verifying that no or only trace levels of target analytes were detected. Sample preparation was performed according to a slightly adjusted dilute and shoot procedure as described by Sulyok et al. [35]. Briefly, $0.500 \pm 0.005 \mathrm{~g}$ of cereal flour was weighed into glass extraction tubes with screw caps. For validation, the blank cereal samples were then spiked with the appropriate aliquot of the final multi-analyte working solution prior to extraction. The spiking levels (Table 1) were chosen to cover available mycotoxin/ modified mycotoxin occurrence data, as well as the legislative limits set for mycotoxins [27, 28]. The samples were allowed to soak overnight in the dark at room temperature. The extraction was performed by adding $2 \mathrm{~mL}$ extraction solution
Table 1 Spiked target analyte concentration levels in barley, oats and wheat blank matrices during validation of the LC-MS/MS method

\begin{tabular}{lrrr}
\hline Analyte & \multicolumn{2}{c}{ Spiking level $(\mu \mathrm{g} / \mathrm{kg})$} & \\
\cline { 2 - 4 } & Low & Medium & High \\
\hline 3Ac-DON & 20 & 80 & 160 \\
DON & 100 & 400 & 800 \\
DON3Glc & 25 & 100 & 200 \\
HT2 & 10 & 50 & 100 \\
HT2-3-Glc & 8 & 30 & 60 \\
NIV & 40 & 80 & 160 \\
NIV3Glc & 20 & 40 & 80 \\
T2 & 10 & 50 & 100 \\
ZEN & 15 & 60 & 120 \\
ZEN14Glc & 8 & 30 & 60 \\
ZEN14Sulf & 8 & 30 & 60 \\
ZEN16Glc & 8 & 30 & 60 \\
$\alpha$-ZEL & 8 & 30 & 60 \\
$\alpha$-ZEL14Glc & 8 & 30 & 60 \\
$\beta-Z E L$ & 8 & 30 & 60 \\
$\beta$-ZEL14Glc & 8 & 30 & 60 \\
\hline
\end{tabular}

(acetonitrile:water:acetic acid, 79:20:1, v/v/v), followed by vortexing (30 s) and mixing in a VKS-75 Control horizontal shaker (Edmund Bühler, Bodelshausen, Germany) at $200 \mathrm{rpm}$ for $90 \mathrm{~min}$. After extraction, the samples were centrifuged at $2700 \times g$ for 10 min with a Heraeus Multifuge S-R centrifuge (Hanau, Germany). Finally, $350 \mu \mathrm{L}$ of the supernatant of each sample was transferred into HPLC vials and diluted with an equal amount of dilution solvent (acetonitrile:water:acetic acid, 20:79:1, v/v/v) and injected into the LC-MS/MS system. For oats, an additional defatting step was employed, where $1 \mathrm{~mL}$ of hexane was added to the extract (ca. $1.2 \mathrm{~mL}$ ) and the mixture was shaken for $10 \mathrm{~min}$, followed by centrifugation at $2700 \times \mathrm{g}$ for $5 \mathrm{~min}$. The hexane layer was removed with a Pasteur pipette, and $350 \mu \mathrm{L}$ of the extract layer was diluted with $350 \mu \mathrm{L}$ dilution solvent and transferred into HPLC vials for analysis. The survey samples were prepared in duplicate using the same process as for the validation samples.

\section{LC-MS/MS analysis}

The samples were analysed using a Waters Acquity ${ }^{\mathrm{TM}}$ UPLC system (Waters Co., Milford, MA, USA) coupled to a Waters $\mathrm{Xevo}^{\mathrm{TM}}$ triple quadrupole (TQ) MS (Micromass, Manchester, UK), equipped with an electrospray ionisation (ESI) interface. A Waters Atlantis ${ }^{\circledR} \mathrm{T} 3$ column $(150 \times 3.0 \mathrm{~mm}, 3.0 \mu \mathrm{m})$ with a matching Atlantis T3 pre-column was used for chromatographic separation, which was carried out at room temperature with a flow rate of $0.35 \mathrm{~mL} / \mathrm{min}$ using binary gradient elution. Eluent A was water buffered with $10 \mathrm{mM}$ ammonium acetate, 
whereas eluent B was acetonitrile. The gradient programme was initiated with a holding time of $2 \mathrm{~min}$ at $5 \% \mathrm{~B}$, after which $55 \%$ B was linearly reached within $5 \mathrm{~min}$. These conditions were maintained for 2 min before a further linear increase of B to $90 \%$ within 6 min and isocratic elution for 2 min. Finally, the starting conditions were switched back (at $17 \mathrm{~min}$ ) followed by a 4-min hold time to allow re-equilibration, resulting in a total run time of $21 \mathrm{~min}$. The injection volume was $5 \mu \mathrm{L}$ and the autosampler was maintained at $10{ }^{\circ} \mathrm{C}$

The ESI source parameters were as follows: capillary voltage, $2.9 \mathrm{kV}$; source temperature, $150{ }^{\circ} \mathrm{C}$; desolvation temperature, $350{ }^{\circ} \mathrm{C}$ and desolvation gas flow, $950 \mathrm{~L} / \mathrm{h}$. Precursor and product ion selection and optimisation of the MS/MS conditions (e.g. cone voltages and collision energies) were conducted using the Waters IntelliStart ${ }^{\mathrm{TM}}$ software by infusion $(10 \mu \mathrm{L} / \mathrm{min})$ of individual analyte standards $(0.5-1.5 \mu \mathrm{g} / \mathrm{mL})$ dissolved in acetonitrile. The MS detection was performed with the selected reaction monitoring (SRM) mode, resulting in automatically optimised dwell times of 22-151 ms. Two SRM transitions were monitored for each compound; the one with the highest intensity was selected as the quantifier ion and the other as the qualifier. The cone voltages and collision energies are presented in Table 2, along with other parameters of the analytes included in this method. Data acquisition and processing were performed with Waters MassLynx ${ }^{\mathrm{TM}}$ v.4.1 and QuanLynx ${ }^{\mathrm{TM}}$ v.4.1 software.

\section{Method validation and data evaluation}

The method was validated for wheat, barley and oats in terms of linearity, specificity, apparent recovery, repeatability, interday precision, the limit of detection (LOD) and the limit of quantification (LOQ), following the performance criteria and recommendations of Commission Regulation EC No 401/ 2006 [30] and Commission Decision EC No 657/2002 [31]. Each of the three concentration levels for validation included six spiked samples for each matrix. Blank barley, oat and wheat samples $(n=20$ per matrix) were also analysed along with the spiked ones. The process was repeated on 3 days. Prior to validation, the efficiency of different extraction solutions was investigated. Furthermore, matrix effects were estimated as the signal suppression and enhancement (SSE) ratio to determine whether the use of matrix-assisted calibration curves in the experiments was necessary. The SSE values were obtained in triplicate as the percentage of the slope of calibration curves prepared in spiked extracts divided by the slope of curves prepared in neat solution [35].

The MS response (peak area) was plotted against the analyte concentration to calculate a linear, $1 / x$ weighted calibration curve. The concentration ranges of the five-point calibration curves were $20-1200 \mu \mathrm{g} / \mathrm{kg}$ for DON and between 10 and $250 \mu \mathrm{g} / \mathrm{kg}$ for $3 \mathrm{Ac}-\mathrm{DON}$, DON3Glc, HT2, NIV, NIV3Glc, T2 and ZEN, with the lowest calibration point based on the LOQ values of each analyte. For $\alpha$ - and $\beta$-ZEL, as well as for all other modified forms, calibration ranges of 5-100 $\mu \mathrm{g} /$ $\mathrm{kg}$ were used. Linearity was evaluated on neat standard and

Table 2 Precursor/product ion pairs and LC-MS/MS parameters in the ESI-SRM mode for detection of the measured analytes (sorted by retention times)

\begin{tabular}{|c|c|c|c|c|c|c|}
\hline Analyte & Retention time (min) & Molecular ion & Precursor ion $(\mathrm{m} / \mathrm{z})$ & $\begin{array}{l}\text { Product ions }(\mathrm{m} / \mathrm{z}) \\
\text { Quantifier, qualifier }\end{array}$ & Cone voltage $(\mathrm{V})$ & Collision energy $(\mathrm{eV})$ \\
\hline NIV3Glc & 6.62 & {$\left[\mathrm{M}+\mathrm{CH}_{3} \mathrm{COO}\right]^{-}$} & 533.3 & $473.3,263.0$ & 24 & 12,24 \\
\hline NIV & 6.76 & {$\left[\mathrm{M}+\mathrm{CH}_{3} \mathrm{COO}\right]^{-}$} & 371.2 & $311.0,281.1$ & 20 & 10,12 \\
\hline DON3Glc & 6.93 & {$\left[\mathrm{M}+\mathrm{CH}_{3} \mathrm{COO}^{-}\right.$} & 517.2 & $457.1,427.1$ & 26 & 14,20 \\
\hline DON & 7.38 & {$[\mathrm{M}+\mathrm{H}]^{+}$} & 297.3 & $249.1,91.0$ & 18 & 10,36 \\
\hline$\beta$-ZEL14Glc & 8.36 & {$[\mathrm{M}-\mathrm{H}]^{-}$} & 481.1 & $319.1,275.1$ & 26 & 14,34 \\
\hline ZEN16Glc & 8.59 & {$\left[\mathrm{M}+\mathrm{NH}_{4}\right]^{+}$} & 498.4 & $319.1,283.2$ & 16 & 14,28 \\
\hline$\alpha$-ZEL14Glc & 8.72 & {$[\mathrm{M}-\mathrm{H}]^{-}$} & 481.3 & $293.2,301.2$ & 50 & 30,34 \\
\hline HT2-3-Glc & 8.95 & {$\left[\mathrm{M}+\mathrm{NH}_{4}\right]^{+}$} & 604.4 & $157.0,105.0$ & 20 & 36,68 \\
\hline 3Ac-DON & 8.98 & {$[\mathrm{M}-\mathrm{H}]^{-}$} & 337.0 & $173.0,307.1$ & 26 & 6,14 \\
\hline ZEN14Glc & 9.22 & {$\left[\mathrm{M}+\mathrm{NH}_{4}\right]^{+}$} & 498.4 & $319.1,283.2$ & 16 & 14,28 \\
\hline ZEN14Sulf & 9.59 & {$[\mathrm{M}-\mathrm{H}]^{-}$} & 397.0 & $317.1,175.0$ & 32 & 22,34 \\
\hline HT2 & 10.44 & {$\left[\mathrm{M}+\mathrm{NH}_{4}\right]^{+}$} & 442.4 & $263.1,215.1$ & 12 & 10,12 \\
\hline$\beta-Z E L$ & 11.59 & {$[\mathrm{M}-\mathrm{H}]^{-}$} & 319.1 & $174.1,159.8$ & 44 & 28,34 \\
\hline$\alpha-Z E L$ & 12.76 & {$[\mathrm{M}-\mathrm{H}]^{-}$} & 319.1 & $159.8,130.0$ & 40 & 28,34 \\
\hline $\mathrm{T} 2$ & 13.29 & {$\left[\mathrm{M}+\mathrm{NH}_{4}\right]^{+}$} & 484.4 & $305.1,185.1$ & 18 & 14,22 \\
\hline ZEN & 14.58 & {$[\mathrm{M}-\mathrm{H}]^{-}$} & 317.1 & $174.9,131.0$ & 40 & 24,28 \\
\hline
\end{tabular}


spiked matrix curves by fitting the data with a linear regression model. The LODs were determined by 20 blank samples per matrix, as the sum of the mean analyte signal and three times the standard deviation (SD). The concentration values for LOD were calculated by comparing the areas with the corresponding concentrations of the lowest spiked levels of the respective calibrant. The LOQ is presented as three times the LOD. Specificity was estimated as the maximum permitted tolerances of relative quantifier/qualifier ion ratios of each target analyte and compared to those obtained in spiked matrix. Moreover, the retention time of the analyte had to match that obtained by the neat standard within a margin of $\pm 2.5 \%$. To calculate the apparent recovery, spiked sample concentrations were measured using the matrix-assisted calibration curves as the percentage of the measured analyte concentration divided by the spiked concentration. Repeatability $\left(\mathrm{RSD}_{\mathrm{r}}\right)$, expressed as relative standard deviation, was determined as the variation within daily results. Similarly, inter-day precision $\left(\mathrm{RSD}_{\mathrm{R}}\right)$ was assessed as the day-to-day variation. Validation data were processed with Microsoft Excel ${ }^{\circledR} 2010$ (Microsoft Co., Redmond, WA, USA). The reported survey sample concentrations were calculated as the mean value of two replicates. For the calculation of the contamination on average, concentrations below the respective LOQ were assigned a value of $L O Q / 2$, whereas values below LOD were treated as non-contaminated.

\section{Results and discussion}

\section{Method development and optimisation}

The method development was initiated by infusing individual analyte solutions into the MS, operating in both positive and negative ESI scan modes. ZEN and its derivatives, with the exception of ZEN14Glc and ZEN16Glc, as well as most of the type $\mathrm{B}$ trichothecene-based analytes showed higher intensities in the negative ESI mode, whereas type A trichothecene-based derivatives were more easily ionised in the positive mode. In order to achieve the best sensitivity for all analytes in the MS using only a single LC run, proper chromatographic separation and frequent polarity switching of the interface during each run was required (Table 2). Initially, a Waters reversedphase $\mathrm{C} 18$ column was tested, but the retention of the more polar modified mycotoxins (e.g. NIV3Glc and DON3Glc) was not satisfactory. Gradient elution adjustments only slightly improved the poor retention of some polar analytes. With the use of the Atlantis column, which is recommended by the manufacturer for the separation of compounds with diverse polarities, significantly better retention for the polar analytes was accomplished and also baseline separation between most isomeric pairs. However, the isomeric compounds ZEN14Glc/ZEN16Glc co-eluted, making them indistinguishable, as they share common transitions. For ZEN14Glc and
ZEN16Glc, a short isocratic elution phase between 7 and $9 \mathrm{~min}$ had to be introduced during the linear gradient programme to achieve chromatographic separation. The retention times of the 16 analytes ranged from $6.62 \mathrm{~min}$ (NIV3Glc) to $14.58 \mathrm{~min}$ (ZEN) and are presented in Fig. 3 in the form of overlayed extracted ion chromatograms.

Before finalising the method, different extraction solutions were assessed for their efficiency in extracting the target analytes. All native mycotoxins together with the modified mycotoxins 3Ac-DON, DON3Glc, $\alpha$-ZEL and $\beta$-ZEL were included in the tests. In these trials, four extraction mixtures were used: (i) acetonitrile:water:acetic acid $(79: 20: 1, v / v / v)$, (ii) acetonitrile:water:formic acid (79:20.9:0.1, v/v/v), (iii) acetonitrile:water $(84: 16, v / v)$ and (iv) methanol:water $(84: 16, v / v)$. During the extraction process, $500 \mu \mathrm{g} / \mathrm{kg}$ of each target analyte was spiked in three replicates for barley and wheat matrices; the process was repeated twice. Our tests verified the applicability of the extraction solution (i), which has been successfully used in multi-mycotoxin methods based on the dilute and shoot approach for type A and B trichothecenes, ZEN and some of their modified forms [24, 25, 36]. In particular, for the native mycotoxins, overall extraction recoveries of more than $90 \%$ were achieved with SD values $\leq 10 \%$. In the case of modified mycotoxins, recoveries with (i) were generally higher compared to those of the other mixtures, except for $\beta$-ZEL, which was extracted at around $80 \%$ (data not shown). The extraction solution (ii) performed better for HT2 (96\%) and NIV (93\%) compared to 90 and $91 \%$ obtained with (i), respectively. Overall, (i) and (ii) yielded similar results, higher than (iii) and (iv) especially for the more polar analytes, but (i) was preferable in matching the chromatographic conditions. Concerning matrix effects, in the three cereal matrices, no severe signal suppression to values more than half of the neat standard was observed for any of the analytes (Table 3 for wheat and in the Electronic Supplementary Material (ESM) Tables S1 and S2 for barley and oats). On the other hand, significant matrix-induced enhancement was

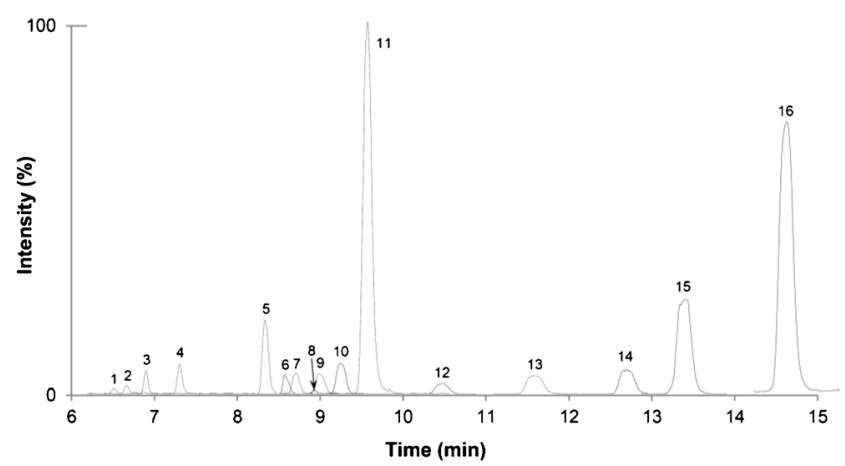

Fig. 3 Overlayed quantifier extracted ion chromatograms of a blank wheat sample spiked at the medium level, with 1 NIV3Glc; 2 NIV; 3 DON3Glc; 4 DON; 5 $\beta$-ZEL14Glc; 6 ZEN16Glc; $7 \alpha$-ZEL14Glc; 8 HT2-3-Glc; 9 3Ac-DON; 10 ZEN14Glc; 11 ZEN14Sulf; 12 HT2; 13 $\beta$-ZEL; $14 \alpha$-ZEL; 15 T2; 16 ZEN 
Table 3 Method performance characteristics and validation parameters determined in wheat

\begin{tabular}{|c|c|c|c|c|c|c|c|c|}
\hline \multirow[t]{2}{*}{ Analyte } & \multirow[t]{2}{*}{ SSE $(\%)$} & \multirow[t]{2}{*}{ LOD $(\mu \mathrm{g} / \mathrm{kg})$} & \multirow[t]{2}{*}{ LOQ $(\mu \mathrm{g} / \mathrm{kg})$} & \multirow[t]{2}{*}{$R^{2}$} & \multirow[t]{2}{*}{ Ion ratio (Quan/Qual) } & \multicolumn{3}{|c|}{ Apparent recovery $\pm \mathrm{RSD}_{\mathrm{R}}(\%)(n=18)$} \\
\hline & & & & & & Low & Medium & High \\
\hline $3 \mathrm{Ac}-\mathrm{DON}$ & 123 & 3.7 & 11.1 & 0.991 & 0.3 & $97 \pm 34$ & $96 \pm 9$ & $100 \pm 10$ \\
\hline DON & 80 & 1.3 & 3.9 & 0.988 & 1.6 & $98 \pm 18$ & $97 \pm 12$ & $99 \pm 8$ \\
\hline DON3Glc & 76 & 2.2 & 6.6 & 0.998 & 1.1 & $99 \pm 10$ & $96 \pm 7$ & $100 \pm 5$ \\
\hline HT2 & 101 & 3.0 & 9.0 & 0.997 & 1.0 & $107 \pm 17$ & $99 \pm 11$ & $101 \pm 9$ \\
\hline HT2-3-Glc & 95 & 3.6 & 10.8 & 0.991 & 0.6 & $108 \pm 26$ & $97 \pm 12$ & $102 \pm 6$ \\
\hline NIV & 67 & 3.2 & 9.6 & 0.986 & 1.4 & $95 \pm 16$ & $93 \pm 8$ & $95 \pm 4$ \\
\hline NIV3Glc & 75 & 4.3 & 12.9 & 0.985 & 4.4 & $100 \pm 21$ & $93 \pm 16$ & $100 \pm 8$ \\
\hline $\mathrm{T} 2$ & 101 & 1.4 & 4.2 & 1.000 & 0.8 & $101 \pm 8$ & $99 \pm 4$ & $101 \pm 4$ \\
\hline ZEN & 82 & 1.9 & 5.7 & 0.998 & 1.3 & $99 \pm 14$ & $98 \pm 4$ & $99 \pm 4$ \\
\hline ZEN14Glc & 92 & 0.1 & 0.3 & 0.997 & 2.5 & $111 \pm 11$ & $96 \pm 8$ & $111 \pm 6$ \\
\hline ZEN14Sulf & 75 & 0.2 & 0.6 & 0.997 & 7.4 & $100 \pm 13$ & $94 \pm 5$ & $100 \pm 5$ \\
\hline ZEN16Glc & 68 & 0.3 & 0.9 & 0.988 & 3.1 & $112 \pm 19$ & $101 \pm 8$ & $112 \pm 8$ \\
\hline$\alpha-Z E L$ & 87 & 0.1 & 0.3 & 0.998 & 1.1 & $96 \pm 13$ & $95 \pm 7$ & $96 \pm 4$ \\
\hline$\alpha$-ZEL14Glc & 92 & 0.5 & 1.5 & 0.994 & 2.3 & $94 \pm 19$ & $95 \pm 10$ & $94 \pm 8$ \\
\hline$\beta-Z E L$ & 84 & 0.3 & 0.9 & 0.997 & 1.1 & $101 \pm 12$ & $94 \pm 6$ & $101 \pm 5$ \\
\hline$\beta$-ZEL14Glc & 86 & 0.2 & 0.6 & 0.997 & 8.7 & $103 \pm 23$ & $96 \pm 25$ & $103 \pm 4$ \\
\hline
\end{tabular}

SSE signal suppression/enhancement ratio, $L O D$ limit of detection, $L O Q$ limit of quantification, $R^{2}$ coefficient of determination, Quan quantifier ion, Qual qualifier ion, $R S D_{R}$ inter-day precision

observed for 3Ac-DON in all three matrices (109-123 \%) and HT2-3-Glc (121\%) in barley. In general, ZEN metabolites were found to have a fluctuating matrix-related behaviour among the different cereals. Therefore, matrix-assisted calibration spiked prior to extraction and prepared identically to the samples was used for quantification of the target analytes, in order to take into account extraction recovery and matrix effects.

\section{Method performance characteristics}

The developed LC-MS/MS method was successfully validated for the determination of 16 analytes (3Ac-DON, DON, DON3Glc, HT2, HT2-3-Glc, NIV, NIV3Glc, T2, ZEN, ZEN14Glc, ZEN14Sulf, ZEN16Glc, $\alpha$-ZEL, $\alpha$-ZEL14Glc, $\beta$-ZEL and $\beta$-ZEL14Glc) in barley, oats and wheat. The method performance characteristics obtained during the validation procedure are presented in Table 3 for wheat and in ESM Tables S1 and S2 for barley and oats, respectively.

LOD values ranged from 0.1 to $4.1 \mu \mathrm{g} / \mathrm{kg}$ in barley, 0.1 to $5.3 \mu \mathrm{g} / \mathrm{kg}$ in oats and 0.1 to $4.3 \mu \mathrm{g} / \mathrm{kg}$ in wheat, allowing the detection of mycotoxins and their modified forms in the low microgram-per-kilogram region. In general, DON, T2 and the derivatives of ZEN had the lowest LODs, and subsequently LOQ values, in all matrices. Among the trichothecene derivatives, the highest LODs were observed for 3Ac-DON, HT2-3Glc and NIV3Glc. Calibration curves were linear over the respective working range of each analyte, with the coefficient of determination $\left(R^{2}\right)$ values being $0.982-1.000$ for the five-point matrix-assisted calibration curves. For those survey samples whose concentrations exceeded the linear range of analytes, appropriate dilutions with blank extracts were performed. Specificity of the target analytes with SRM was confirmed by the presence of analyte quantifier/qualifier transitions at the correct retention times in neat standard. This parameter was also verified by the absence of any interfering peaks in the SRM channels in the 20 blank samples analysed per matrix.

Accuracy and precision were evaluated by recovery experiments. The apparent recoveries varied between 90 and $116 \%$ in barley, 84 and $115 \%$ in oats and 93 and $112 \%$ in wheat for all analytes. For those analytes for which performance criteria were available, the apparent recoveries were in agreement with the recommended legislative values (typically 70-120 \%). For compounds for which no such values exist, acceptable apparent recovery was also achieved. In the case of NIV in oats, two outliers were removed from the low level because of a spiking error. The matrix effects (expressed as SSE) ranged between 59 and $121 \%$ for barley, 53 and $122 \%$ for oats and 67 and $123 \%$ for wheat. Based on these results, it was proven that the extraction solution (acetonitrile:water:acetic acid, 79:20:1, v/v/v) uniformly suited all analytes included in the method. $\mathrm{RSD}_{\mathrm{r}}$ and $\mathrm{RSD}_{\mathrm{R}}$ were also calculated for the three concentration levels, but only the $\mathrm{RSD}_{\mathrm{R}}$ values are presented here. The $\mathrm{RSD}_{\mathrm{r}}$ values were relatively high for the glucosylated NIV and HT2 derivatives but never exceeded though the $40 \%$ limit set in EC No 401/2006 for the native HT2 at the low concentration level 


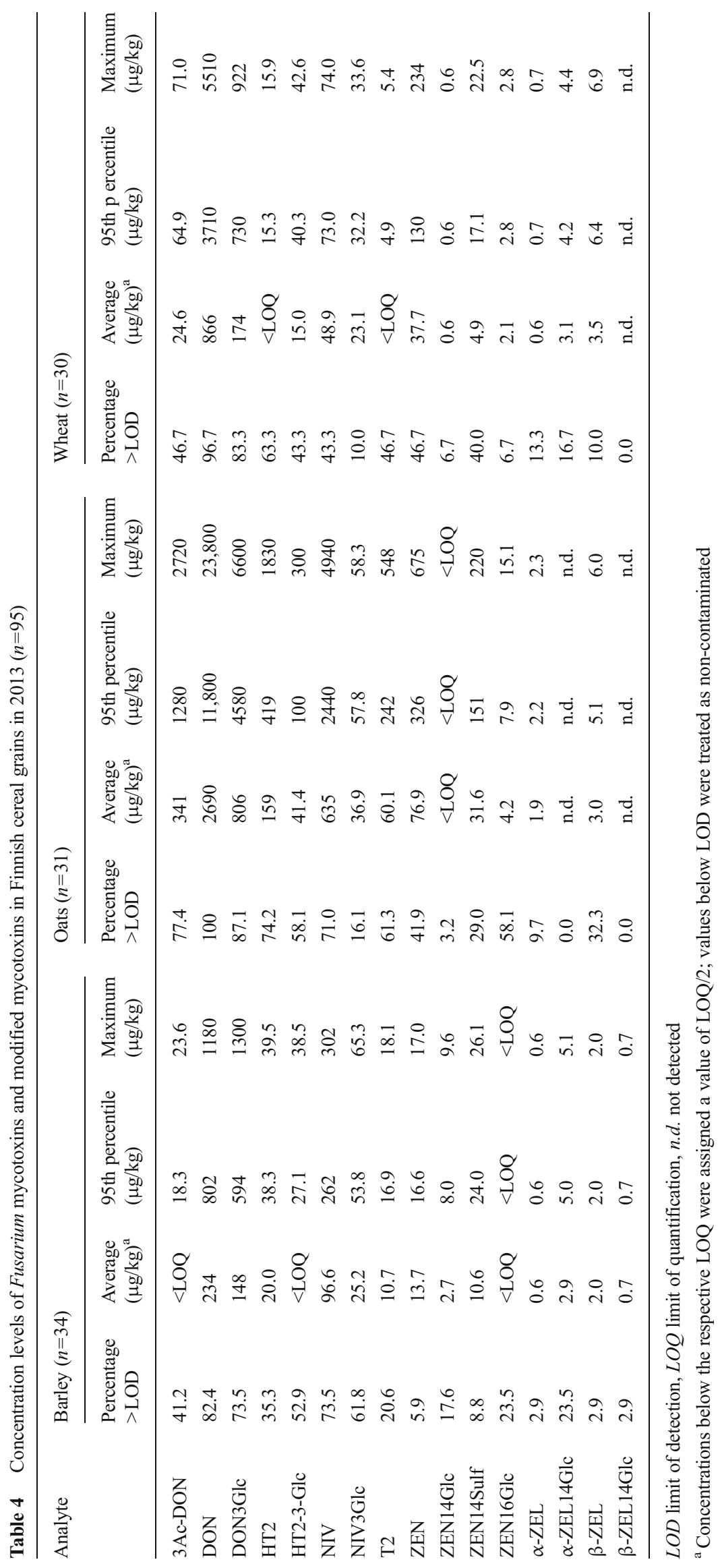


$\left(100-200 \mu \mathrm{g} / \mathrm{kg}\right.$ ) [30]. Finally, $\mathrm{RSD}_{\mathrm{R}}$ values were below the $40-60 \%$ limit required by the same regulation for analysis of the native DON, HT2, T2 and ZEN, as no performance criteria are currently available for modified forms.

\section{Naturally contaminated raw cereal samples}

The developed and validated LC-MS/MS method was applied for the analysis of 95 cereal grain samples from Finland harvested during 2013, and the results are summarised in Table 4. Figure 4 presents an overview of the detected mycotoxins and modified mycotoxins in barley, oats and wheat. The results of our survey revealed considerable variation between different commodities. The highest contamination of the studied mycotoxins was observed in oats, followed by wheat and barley. The most frequently detected native mycotoxin was DON (incidence $93 \%$ of the samples analysed), followed by NIV (63\%) and HT2 (57\%). DON was also found at the highest concentrations, and particularly in wheat and oats, with $32 \%$ of the analysed oats exceeding the legislative ML of $1750 \mu \mathrm{g} /$ $\mathrm{kg}$ set for DON regarding unprocessed oats placed on the market for first-stage processing [27]. For wheat, the percentage of samples exceeding the ML $(1250 \mu \mathrm{g} / \mathrm{kg})$ was $23 \%$, whereas no barley sample exceeded the same level. 3Ac-DON $(55 \%)$ was only found at increased levels in oats, in samples also showing high concentrations of DON. One wheat and one oat sample also exceeded the ML set for ZEN $(100 \mu \mathrm{g} /$ $\mathrm{kg}$ ), and both were also highly contaminated with DON (1660 and $23,800 \mu \mathrm{g} / \mathrm{kg}$, respectively). Such DON levels are unusually high compared to those of previous years based on quantitative results from the Finnish grain quality monitoring programme (1999-2009) [9]. Only minor amounts of HT2 and $\mathrm{T} 2$ were detected in barley and wheat. In contrast, the prevalence of these toxins in oats was significantly more pronounced. Nonetheless, only one sample exceeded the indicative value for the sum of these toxins $(1000 \mu \mathrm{g} / \mathrm{kg})$ in Recommendation 2013/165/EU for unprocessed oats [28]. NIV contamination was relatively low in barley and wheat samples in comparison to the levels found in oats, with a mean concentration of $635 \mu \mathrm{g} / \mathrm{kg}$ that can be attributed to four very highly contaminated samples (>1400 $\mu \mathrm{g} / \mathrm{kg})$.

Apart from the overall high DON levels and a few oats with high NIV concentrations, the prevalence and contamination levels of all other native mycotoxins investigated in this study were well in accordance with the previous survey results from Finland [9]. Oats appear to be the most susceptible commodity, with high mycotoxin levels, especially DON and HT2/T2. Mostly oats, but also some barley and wheat samples from areas 4, 7-10 and 11 (Fig. 2), were contaminated with the highest DON concentrations. In those areas, located in the central and eastern parts of Finland, rainfall occurred during the flowering period of oats, i.e. around the end of June and beginning of July, followed by warm weather, conditions optimal for DON production. Until recently, the dominant DONproducing species in Finland was Fusarium culmorum in barley and wheat, but during the past few years, it has been replaced by Fusarium graminearum, the populations of which are rapidly spreading not only in Finland but also in other northern countries [37]. As F. graminearum is a more prolific DON producer than F. culmorum, it may have contributed to the high DON concentrations. In the case of HT2 and T2, the most contaminated samples were from the southern parts of Finland, which had a drier and warmer climate more appropriate for the major HT2/T2 producers in Finnish oats (Fusarium sporotrichioides and Fusarium langsethiae). NIV is mostly produced by Fusarium poae and in smaller amounts by F. culmorum and F. graminearum in Finland [38]. The generally low concentration levels of NIV in samples with high DON/3Ac-DON contamination support the fact that Finnish F. culmorum and F. graminearum strains are predominantly of
Fig. 4 Overview of the Fusarium mycotoxins and modified mycotoxins detected in Finnish cereal grains in $2013(n=95)$

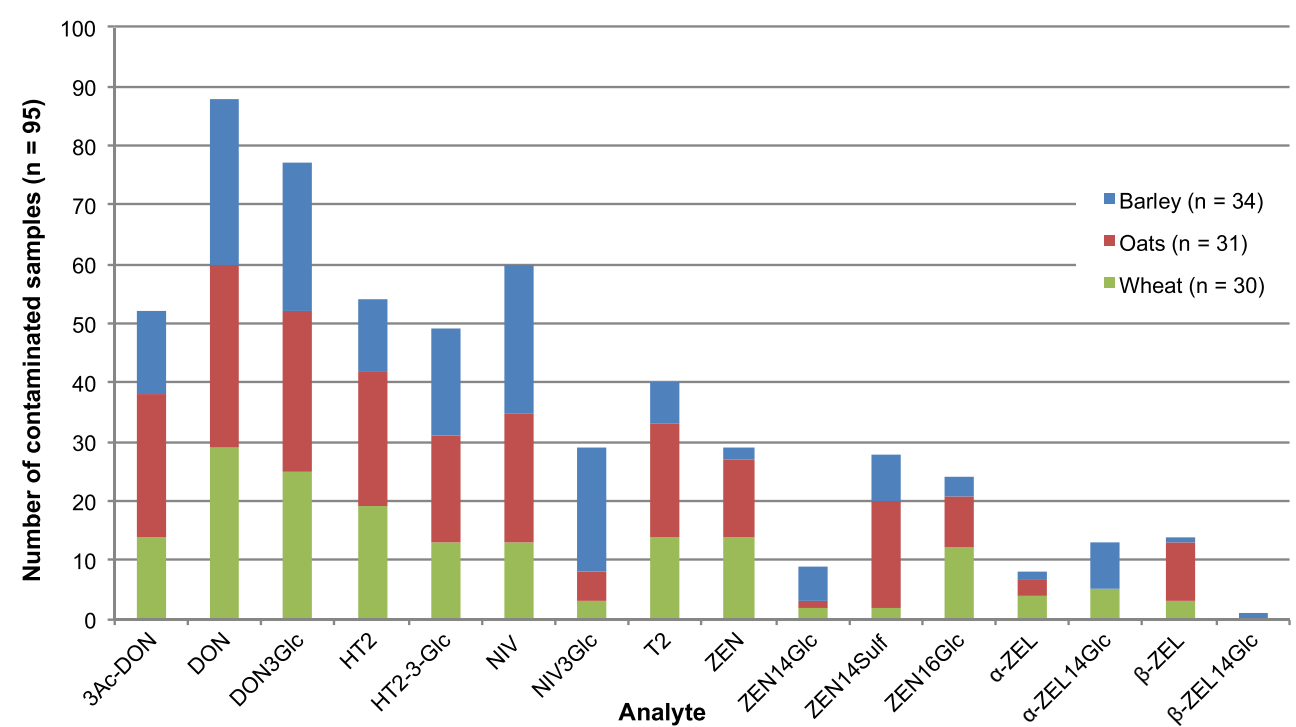


the 3Ac-DON-producing chemotype IA [37]. Finally, ZEN is known to be produced at the end of the growing period before harvesting [39], and the weather at that time was mostly warm and dry in Finland, conditions not favourable for ZEN production, resulting in low to medium levels of contamination.

In this paper, the natural occurrence of some modified mycotoxins (e.g. ZEN16Glc and NIV3Glc) is for the first time presented in some of the commodities analysed here. All of the modified mycotoxins included in the method were quantifiable, with concentrations higher than the respective LOQ of each analyte in at least one sample. DON3Glc $(81 \%)$ was by far the most abundant, with the maximum concentrations reaching $6600 \mu \mathrm{g} / \mathrm{kg}$ in a highly DON-contaminated oat sample. Among the other modified mycotoxins, HT2-3-Glc and NIV3Glc were found in 52 and $31 \%$ of the samples, respectively. The modified/native relative proportion, expressed as a molar ratio, was generally in the range of $15-40 \%$ for DON3Glc/DON and slightly higher for HT2-3-Glc/HT2 $(30-55 \%)$. The former is in line with previous publications showing a stable relative proportion of DON3Glc to DON at around $20 \%[24,40]$. For the latter, no information is currently available in the literature. However, in both cases, barley showed the highest relative proportions of the modified mycotoxins DON3Glc and HT2-3-Glc, and in a few barley samples, the DON3Glc/DON ratio reached levels between 65 and $90 \%$. Notably, according to our findings, the recently reported ZEN16Glc was detected in more samples than the wellknown ZEN14Glc, highlighting the fact that there can still be many modified mycotoxins of significance that may remain unknown. Finally, ZEN14Sulf (29 \%) and ZEN16Glc (25\%) were the most prevalent ZEN metabolites overall, while the other ZEN derivatives (ZEN14Glc, $\alpha$-ZEL, $\alpha$ ZEL14Glc, $\beta$-ZEL and $\beta$-ZEL14Glc) were found in less than $15 \%$ of the samples and at low levels.

\section{Conclusions}

This article describes the development and validation of a multi-mycotoxin LC-MS/MS method for the simultaneous analysis of Fusarium mycotoxins, including major type A and $\mathrm{B}$ trichothecenes and $\mathrm{ZEN}$, as well as certain modified forms in cereals. The method was in-house validated in barley, oats and wheat. Rapid and reliable detection of the target analytes was achieved with this method, offering the ability to obtain quantitative data for modified mycotoxins along with their native forms in cereals during a single run. By utilising this method, we were able for the first time to present occurrence data for a number of modified mycotoxins from cereal grains cultivated in Finland. All of the modified mycotoxins analysed with this method could be detected in the naturally contaminated survey samples, with the most prevalent being DON3Glc, which was also found in the highest concentrations, followed by HT2-3-Glc and NIV3Glc. Regarding native mycotoxins, the contamination levels of DON, NIV and HT2 were the highest, particularly in oats, with their distribution affected by favourable climatic conditions in particular areas for Fusarium mycotoxin production.

Acknowledgments This study was co-funded by the Finnish Funding Agency for Technology and Innovation (TEKES; Myco-DETECT project, 401/31/2011). The Vienna Science and Technology Fund (WWTF LS12-012) and the Austrian Science Fund (FWF, SFB Fusarium, F3708 and F3706) funded the projects to produce the mycotoxin glucosides used in this study. The Austrian Federal Ministry of Science, Research and Economy, the Austrian National Foundation for Research, Technology and Development as well as BIOMIN Holding GmbH and Nestec Ltd. are gratefully acknowledged for funding the Christian Doppler Laboratory for Mycotoxin Metabolism. The Lower Austrian Government supported A.M. and E.V. The authors would like to thank Eija Hyvönen for her assistance in the laboratory, as well as Pia Vilen and Anne Mäittälä for their help in the Finnish map design.

Open Access This article is distributed under the terms of the Creative Commons Attribution 4.0 International License (http://creativecommons.org/ licenses/by/4.0/), which permits unrestricted use, distribution, and reproduction in any medium, provided you give appropriate credit to the original author(s) and the source, provide a link to the Creative Commons license, and indicate if changes were made.

\section{References}

1. Bottalico A, Perrone G (2002) Toxigenic Fusarium species and mycotoxins associated with head blight in small-grain cereals in Europe. Eur J Plant Pathol. doi:10.1023/A:1020635214971

2. Grove JF (2007) The trichothecenes and their biosynthesis. Prog Chem Org Nat Prod 88:63-130

3. McCormick SP, Stanley AM, Stover NA, Alexander NJ (2011) Trichothecenes: from simple to complex mycotoxins. Toxins. doi: 10.3390/toxins 3070802

4. Parent-Massin D (2004) Haematotoxicity of trichothecenes. Toxicol Lett. doi:10.1016/j.toxlet.2004.04.024

5. Pestka JJ (2010) Deoxynivalenol: mechanisms of action, human exposure, and toxicological relevance. Arch Toxicol. doi:10.1007/ s00204-010-0579-8

6. Yli-Mattila T (2011) Detection of trichothecene-producing Fusarium species in cereals in Northern Europe and Asia. Agron Res 9:521-526

7. Edwards SG (2009) Fusarium mycotoxin content of UK organic and conventional oats. Food Addit Contam A. doi:10.1080/ 02652030902788953

8. Pettersson H, Brown C, Hauk J, Hoth S, Meyer J, Wessels D (2011) Survey of T-2 and HT-2 toxins by LC-MS/MS in oats and oat products from European oat mills in 2005-2009. Food Addit Contam B. doi:10.1080/19393210.2011.561933

9. Van Der Fels-Klerx HJ, Klemsdal S, Hietaniemi V, Lindblad M, Ioannou-Kakouri E, Van Asselt ED (2012) Mycotoxin contamination of cereal grain commodities in relation to climate in North West Europe. Food Addit Contam A. doi:10.1080/19440049.2012. 689996 
10. Metzler M, Pfeiffer E, Hildebrand A (2010) Zearalenone and its metabolites as endocrine disrupting chemicals. World Mycotoxin J. doi:10.3920/WMJ2010.1244

11. EFSA Panel on Contaminants in the Food Chain (CONTAM) (2011) Scientific opinion on the risks for public health related to the presence of zearalenone in food. EFSA Journal 9(6):2197

12. Rychlik M, Humpf H, Marko D, Dänicke S, Mally A, Berthiller F, Klaffke H, Lorenz N (2014) Proposal of a comprehensive definition of modified and other forms of mycotoxins including "masked" mycotoxins. Mycotoxin Res. doi:10.1007/s12550-014-0203-5

13. Berthiller F, Crews C, Dall'Asta C, Saeger SD, Haesaert G, Karlovsky P, Oswald IP, Seefelder W, Speijers G, Stroka J (2013) Masked mycotoxins: a review. Mol Nutr Food Res. doi:10.1002/ mnfr.201100764

14. Sewald N, von Gleissenthall JL, Schuster M, Müller G, Aplin RT (1992) Structure elucidation of a plant metabolite of 4desoxynivalenol. Tetrahedron Asymmetry. doi:10.1016/S09574166(00)82193-X

15. Busman M, Poling SM, Maragos CM (2011) Observation of T-2 toxin and HT-2 toxin glucosides from Fusarium sporotrichioides by liquid chromatography coupled to tandem mass spectrometry (LC-MS/MS). Toxins (Basel). doi:10.3390/toxins3121554

16. Nakagawa H, Ohmichi K, Sakamoto S, Sago Y, Kushiro M, Nagashima H, Yoshida M, Nakajima T (2011) Detection of a new Fusarium masked mycotoxin in wheat grain by high-resolution LC-Orbitrap ${ }^{\mathrm{TM}}$ MS. Food Addit Contam A. doi:10.1080/ 19440049.2011.597434

17. Berthiller F, Werner U, Sulyok M, Krska R, Hauser M, Schuhmacher R (2006) Liquid chromatography coupled to tandem mass spectrometry (LC-MS/MS) determination of phase II metabolites of the mycotoxin zearalenone in the model plant Arabidopsis thaliana. Food Addit Contam. doi:10.1080/02652030600778728

18. Kovalsky Paris MP, Schweiger W, Hametner C, Stuckler R, Muehlbauer GJ, Varga E, Krska R, Berthiller F, Adam G (2014) Zearalenone-16-O-glucoside: a new masked mycotoxin. J Agric Food Chem. doi:10.1021/jf405627d

19. Dall'Erta A, Cirlini M, Dall'Asta M, Del Rio D, Galaverna G, Dall'Asta C (2013) Masked mycotoxins are efficiently hydrolyzed by human colonic microbiota releasing their aglycones. Chem Res Toxicol. doi:10.1021/tx300438c

20. Nagl V, Woechtl B, Schwartz-Zimmermann HE, Hennig-Pauka I, Moll W, Adam G, Berthiller F (2014) Metabolism of the masked mycotoxin deoxynivalenol-3-glucoside in pigs. Toxicol Lett. doi: 10.1016/j.toxlet.2014.06.032

21. Pereira V, Fernandes J, Cunha S (2014) Mycotoxins in cereals and related foodstuffs: a review on occurrence and recent methods of analysis. Trends Food Sci Technol. doi:10.1016/j.tifs.2014.01.005

22. Berthiller F, Schuhmacher R, Adam G, Krska R (2009) Formation, determination and significance of masked and other conjugated mycotoxins. Anal Bioanal Chem. doi:10.1007/s00216-009-2874-x

23. Cirlini M, Dall'Asta C, Galaverna G (2012) Hyphenated chromatographic techniques for structural characterization and determination of masked mycotoxins. J Chromatogr A. doi:10.1016/j.chroma. 2012.02.057

24. De Boevre M, Di Mavungu JD, Maene P, Audenaert K, Deforce D, Haesaert G, Eeckhout M, Callebaut A, Berthiller F, Van Peteghem C, De Saeger S (2012) Development and validation of an LC-MS/ MS method for the simultaneous determination of deoxynivalenol, zearalenone, T-2-toxin and some masked metabolites in different cereals and cereal-derived food. Food Addit Contam A. doi:10. 1080/19440049.2012.656707

25. Varga E, Glauner T, Berthiller F, Krska R, Schuhmacher R, Sulyok M (2013) Development and validation of a (semi-) quantitative UHPLC-MS/MS method for the determination of 191 mycotoxins and other fungal metabolites in almonds, hazelnuts, peanuts and pistachios. Anal Bioanal Chem. doi:10.1007/s00216-013-6831-3
26. Nathanail AV, Sarikaya E, Jestoi M, Godula M, Peltonen K (2014) Determination of deoxynivalenol and deoxynivalenol-3-glucoside in wheat and barley using liquid chromatography coupled to mass spectrometry: on-line clean-up versus conventional sample preparation techniques. J Chromatogr A. doi:10.1016/j.chroma.2014.11.046

27. Commission Regulation (EC) No 1881/2006 of 19 December 2006 setting maximum levels for certain contaminants in foodstuffs (2006) Off J Eur Union L 364:5

28. Commission Recommendation 2013/165/EU of 27 March 2013 on the presence of T-2 and HT-2 toxin in cereals and cereal products (2013) Off J Eur Commun L 91:12

29. EFSA Panel on Contaminants in the Food Chain (CONTAM) (2014) Scientific opinion on the risks for human and animal health related to the presence of modified forms of certain mycotoxins in food and feed. EFSA J 12(12):3916

30. Commission Regulation (EC) No 401/2006 of 23 February 2006 laying down the methods of sampling and analysis for the official control of the levels of mycotoxins in foodstuffs (2006) Off J Eur Union L 70:12

31. Commission Decision 2002/657/EC of 12 August 2002 on implementing Council Directive 96/23/EC concerning the performance of analytical methods and the interpretation of results (2002) Off J Eur Commun L 221:8

32. Berthiller F, Dall'Asta C, Schuhmacher R, Lemmens M, Adam G, Krska R (2005) Masked mycotoxins: determination of a deoxynivalenol glucoside in artificially and naturally contaminated wheat by liquid chromatography-tandem mass spectrometry. $\mathrm{J}$ Agric Food Chem. doi:10.1021/jf047798g

33. Berthiller F, Hametner C, Krenn P, Schweiger W, Ludwig R, Adam G, Krska R, Schuhmacher R (2009) Preparation and characterization of the conjugated Fusarium mycotoxins zearalenone-4O- $\beta$-Dglucopyranoside, $\alpha$-zearalenol-4O- $\beta$-D-glucopyranoside and $\beta$ zearalenol-4O- $\beta$-D-glucopyranoside by MS/MS and two-dimensional NMR. Food Addit Contam A. doi:10.1080/02652030802399034

34. Mikula H, Sohr B, Skrinjar P, Weber J, Hametner C, Berthiller F, Krska R, Adam G, Fröhlich J (2013) Sulfation of $\beta$-resorcylic acid esters - first synthesis of zearalenone-14-sulfate. Tetrahedron Lett. doi:10.1016/j.tetlet.2013.04.059

35. Sulyok M, Berthiller F, Krska R, Schuhmacher R (2006) Development and validation of a liquid chromatography/tandem mass spectrometric method for the determination of 39 mycotoxins in wheat and maize. Rapid Commun Mass Spectrom. doi:10.1002/rcm.2640

36. Malachová A, Sulyok M, Beltrán E, Berthiller F, Krska R (2014) Optimization and validation of a quantitative liquid chromatography-tandem mass spectrometric method covering 295 bacterial and fungal metabolites including all regulated mycotoxins in four model food matrices. J Chromatogr A. doi:10.1016/j.chroma. 2014.08.037

37. Yli-Mattila T (2010) Ecology and evolution of toxigenic Fusarium species in cereals in northern Europe and Asia. J Plant Pathol 92(1): 7-18

38. Yli-Mattila T, Paavanen-Huhtala S, Jestoi M, Parikka P, Hietaniemi V, Gagkaeva T, Sarlin T, Haikara A, Laaksonen S, Rizzo A (2008) Real-time PCR detection and quantification of Fusarium poae, $F$. graminearum, F. sporotrichioides and F. langsethiae in cereal grains in Finland and Russia. Arch Phytopathology Plant Protect. doi:10.1080/03235400600680659

39. Matthäus K, Dänicke S, Vahjen W, Simon O, Wang J, Valenta H, Meyer K, Strumpf A, Ziesenib H, Flachowsky G (2004) Progression of mycotoxin and nutrient concentrations in wheat after inoculation with Fusarium culmorum. Arch Anim Nutr. doi:10. $1080 / 00039420310001656668$

40. Desmarchelier A, Seefelder W (2011) Survey of deoxynivalenol and deoxynivalenol-3-glucoside in cereal-based products by liquid chromatography electrospray ionization tandem mass spectrometry. World Mycotoxin J. doi:10.3920/WMJ2010.1236 